\title{
Impact of 27-hydroxylase (CYP27A1) and 27-hydroxycholesterol in breast cancer
}

\author{
Siker Kimbung', Ching-yi Chang'2, Pär-Ola Bendahl', Laura Dubois3, \\ J Will Thompson 2,3, Donald P McDonnell2 and Signe Borgquist ${ }^{1,4}$
}

1Division of Oncology and Pathology, Department of Clinical Sciences, Lund, Lund University, Sweden 2Department of Pharmacology and Cancer Biology, Duke University School of Medicine, Durham, NC, USA 3Duke Proteomics and Metabolomics Resource, Duke University School of Medicine, Durham, NC, USA ${ }^{4}$ Clinical Trial Unit, Clinical Studies Sweden, Forum South, Skåne University Hospital, Lund, Sweden
Correspondence should be addressed to S Kimbung or S Borgquist Email

siker.kimbung@med.lu.se or signe.borgquist@med.lu.se

\begin{abstract}
The impact of systemic 27-hydroxycholesterol $(27 \mathrm{HC})$ and intratumoral CYP27A1 expression on pathobiology and clinical response to statins in breast cancer needs clarification. $27 \mathrm{HC}$ is an oxysterol produced from cholesterol by the monooxygenase CYP27A1, which regulates intracellular cholesterol homeostasis. $27 \mathrm{HC}$ also acts as an endogenous selective estrogen receptor (ER) modulator capable of increasing breast cancer growth and metastasis. $27 \mathrm{HC}$ levels can be modulated by statins or direct inhibition of CYP27A1, thereby attenuating its pro-tumorigenic activities. Herein, the effect of statins on serum 27HC and tumor-specific CYP27A1 expression was evaluated in 42 breast cancer patients treated with atorvastatin within a phase II clinical trial. Further, the associations between CYP27A1 expression with other primary tumor pathological features and clinical outcomes were studied in two additional independent cohorts. Statin treatment effectively decreased serum $27 \mathrm{HC}$ and deregulated CYP27A1 expression in tumors. However, these changes were not associated with anti-proliferative responses to statin treatment. CYP27A1 was heterogeneously expressed among primary tumors, with high expression significantly associated with high tumor grade, ER negativity and basal-like subtype. High CYP27A1 expression was independently prognostic for longer recurrence-free and overall survival. Importantly, the beneficial effect of high CYP27A1 in ER-positive breast cancer seemed limited to women aged $\leq 50$ years. These results establish a link between CYP27A1 and breast cancer pathobiology and prognosis and propose that the efficacy of statins in reducing serum lipids does not directly translate to anti-proliferative effects in tumors. Changes in other undetermined serum or tumor factors suggestively mediate the anti-proliferative effects of statins in breast cancer.
\end{abstract}

Endocrine-Related Cancer (2017) 24, 339-349

\section{Background}

Obesity and hypercholesterolemia are associated with an increased risk of developing estrogen receptoralpha $(\mathrm{ER} \alpha)$-positive breast cancers, especially among postmenopausal women (Boyd \& Mcguire 1990, Bianchini et al. 2002, Kitahara et al. 2011), although the molecular factors linking these phenotypes to breast http://erc.endocrinology-journals.org DOI: 10.1530/ERC-16-0533
C 2017 The authors Published by Bioscientifica Ltd. Printed in Great Britain

\section{Key Words}

- 27-hydroxycholesterol

- CYP27A1

- breast cancer

- statin 
cancer etiology and progression remain elusive. Recently, it was demonstrated that 27-hydroxycholesterol (27HC), an oxysterol produced by the sterol 27-hydroxylase cytochrome P450 27A1 (CYP27A1) from cholesterol, was a bona fide endogenous selective estrogen receptor modulator (SERM) that manifested partial agonist activity in ERo-positive breast cancer cells (Umetani et al. 2007, DuSell et al. 2008). As such, it was not surprising that 27HC was shown to increase breast cancer growth and metastatic progression in preclinical experimental models and that this activity was attenuated by genetic and pharmacological approaches (i.e. inhibition of either 3-hydroxy-3-methylglutaryl-CoA reductase (HMGCR)) that interfered with the production of cholesterol and/or the conversion of cholesterol to 27HC (Nelson et al. 2013, $\mathrm{Wu}$ et al. 2013). Thus, although the hydroxylation of cholesterol catalyzed by CYP27A1 is a mechanism utilized by cells to eliminate cholesterol from peripheral tissues, it can also result in the production of an ER ligand that likely contributes to breast cancer pathogenesis.

Despite the substantial amount of preclinical evidence supporting a role for $27 \mathrm{HC}$ in breast cancer pathobiology (Nelson et al. 2013), it remains to be determined if variability in serum and/or intratumoral levels of $27 \mathrm{HC}$ or of CYP27A1 influences clinical breast cancer tumor pathology and patient outcome. The circulating levels of 27HC and cholesterol have been reported to be significantly positively correlated, and plasma $27 \mathrm{HC}$ tends to be elevated with hypercholesterolemia and increasing age (Brown \& Jessup 1999, Burkard et al. 2007, Wu et al. 2013). In general, high CYP27A1 expression is a characteristic of hepatic tissue and stromal cells (macrophages), but differential expression of CYP27A1 has also been shown in breast cancer, where increased tumor cell-specific CYP27A1 expression was more frequently seen among high-grade tumors (Nelson et al. 2013). In addition, the concentration of $27 \mathrm{HC}$ was found to be higher in ERopositive breast tumors compared to normal breast tissue, and this was attributed to a corresponding decrease in the expression of the oxysterol $7 \alpha$-hydroxylase (CYP7B1), the enzyme responsible for metabolizing $27 \mathrm{HC}$ in breast tumors (Wu et al. 2013). The extent to which increased systemic vs intratumoral $27 \mathrm{HC}$ and/or variability in the expression of CYP27A1 expression impacts breast tumor biology has not been evaluated. The limited scope of previous studies addressing this question warrants the conduct of a more comprehensive investigation of the role of CYP27A1 in breast cancer within larger cohorts of patients for which data on conventional prognostic and treatment predictive tumor pathological and molecular features are available.

The growth of most luminal breast cancers is dependent on ER $\alpha$ signaling and on pathways/processes downstream of this receptor. Thus, endocrine therapies, which interfere with the production of extra-gonadal estrogens or which competitively inhibit ER $\alpha$ activation, remain the cornerstone of therapy in postmenopausal breast cancer. However, despite the generally positive effects of aromatase inhibitors, the preferred frontline endocrine intervention in ERo-positive breast cancer, the relatively rapid development of resistance remains an impediment to durable clinical responses. This suggests that other estrogen-independent but possibly ERo-mediated mechanisms, which are unaffected by aromatase inhibitors, may still play key roles in driving tumor progression. Considering its activity in preclinical models of breast cancer, it is likely that increased intratumoral 27HC, could bypass the inhibitory effect(s) of existing aromatase inhibitors (Nelson et al. 2013). Furthermore, because 27HC is a SERM that manifests partial agonist activity in the breast, it is possible that it could attenuate ER $\alpha$ action in pre-menopausal women (Umetani et al. 2007, DuSell et al. 2008). Thus, although high 27HC stimulates ER transcriptional activity under hypo-estrogenic conditions (i.e. among postmenopausal women), the opposite effect may occur under physiologic or high estrogenic conditions (i.e. in pre-menopausal women), where 27HC exhibits ER antagonist activity (Umetani et al. 2007, DuSell et al. 2008, Nelson et al. 2013). This suggests that this cholesterol metabolite may play opposing roles in the pre- and postmenopausal settings. Defining how menopausal status influences the relationship between the systemic or tissue-specific levels of 27HC and CYP27A1 and breast cancer tumor pathological features and patient survival remains to be addressed.

Statins are very effective treatments for hypercholesterolemia, which reduce the circulating levels of both low-density lipoprotein (LDL) cholesterol and total cholesterol through their actions on hepatic HMGCR, the rate-limiting enzyme in cholesterol biosynthesis (Clendening \& Penn 2012). In breast cancer, the cholesterol-lowering effect of statin treatment was shown to prolong tumor latency and inhibit tumor growth in experimental models of ER $\alpha$-positive disease (Nelson et al. 2013). Furthermore, statins have been shown to induce apoptosis in vitro (Spampanato et al. 2012, Bjarnadottir et al. 2015) and in clinical breast cancer 
(Garwood et al. 2010, Bjarnadottir et al. 2015). These data partially provide a molecular explanation for the clinical observation that statins reduce breast cancer recurrence and prolong overall survival in breast cancer patients (Ahern et al. 2011, Chae et al. 2011, Boudreau et al. 2014, Borgquist et al. 2016). Because statins effectively decrease plasma cholesterol in breast cancer patients (Garwood et al. 2010, Higgins et al. 2012), it has been inferred that a commensurate lowering of plasma $27 \mathrm{HC}$ would also occur. Further, given the established roles of $27 \mathrm{HC}$ in breast cancer, it has been inferred that it is the ability of statins to lower $27 \mathrm{HC}$, and not cholesterol per se, that is responsible for the beneficial effects of this class of drugs in patients with ER $\alpha$-positive tumors (McDonnell et al. 2014). Although important to show that systemic levels of $27 \mathrm{HC}$ are in fact lowered by statin treatment in breast cancer patients, it is also of importance to assess the impact of statin treatment on intratumoral 27HC and how this is influenced by CYP27A1 expression in tumors, and how these biochemical activities influence other pathological features of tumors.

The objective of this study was to investigate the impact of statin treatment on serum 27HC and how this correlates with total cholesterol and tumor pathological factors. Further, we aimed to assess tumor cell-specific expression of CYP27A1 in primary breast tumors from patients treated with statins as an anti-cancer agent in a pre-surgical clinical trial. In addition, we present an exploratory analysis of the impact of the heterogeneous expression of CYP27A1, measured at the transcriptional level and by immunohistochemistry, on primary breast cancer regarding clinical and pathological features and prognosis.

\section{Materials and methods}

\section{Patients and tumors}

Three independent cohorts of patients with primary breast cancer were used to test the specific hypotheses of the current investigation.

Cohort 1 This cohort comprises 42 women with primary breast cancer, who were enrolled in a pre-surgical window-of-opportunity trial conducted at the Skåne University Hospital in Lund, Sweden, evaluating the effect of two weeks of high-dose atorvastatin on breast cancer proliferation (Bjarnadottir et al. 2013). The study was registered at ClinicalTrials.gov (\#NCT00816244) December 30, 2008. All patients signed informed written consent.
The present sub-study aimed to investigate the impact of statin treatment on serum 27HC and on tumor-specific CYP27A1 expression. Patients and tumor characteristics at baseline are reported in Supplementary Table 1 (see section on supplementary data given at the end of this article). This cohort was enriched with older women (83\% older than 50 years) presenting with primary tumors that were mostly ER $\alpha$ positive (88\%) and of lower histological grade (62\% grades 1 and 2$)$. In addition, the majority of patients (59\%) presented with positive lymph node status at the time of diagnosis. Serum samples collected before and after the completion of two weeks treatment with high-dose atorvastatin ( $80 \mathrm{mg}$ daily) were analyzed for total cholesterol, LDL cholesterol and high-density lipoprotein (HDL) cholesterol concentrations according to standard clinical diagnostic procedures at Skåne University Hospital, Lund, Sweden. Other tumor pathological factors have been previously assessed (Supplementary Table 1 and Bjarnadottir et al. (2013)). Serum 27HC and tumorspecific CYP27A1 expression were determined by mass spectrometry and immunohistochemistry, respectively. Paired microarray data from a subset of 25 patients (GSE63427, Bjarnadottir et al. (2015)) were also available to enable an evaluation of the impact of statin treatment on CYP27A1 mRNA expression.

Cohort 2 This cohort included 1,881 women with primary breast cancer that was diagnosed and treated at several different institutions worldwide and was used to investigate if heterogeneity in CYP27A1 expression correlated with conventional breast cancer tumor pathological features and prognosis. Gene expression data and the corresponding clinical and tumor pathological data were downloaded from the gene expression omnibus (GEO, 11 independent datasets), and the 11 datasets were merged and normalized to obtain a single mega dataset as previously described (Ringner et al. 2011). A summary of the distribution of tumor pathological factors in this cohort is presented in Supplementary Table 1. The median follow-up for recurrence-free survival (RFS) and overall survival (OS) were 7.2 (range 0.17-24) years and 8.3 (range $0.13-25$ ) years, respectively.

Cohort 3 The results from the analyses performed within cohort 2 were independently verified in a subset of 661 invasive breast carcinomas from the TCGA project (update September 2013). RNAseq v2 level 3 data for the 661 tumors were processed as previously described (Cancer Genome Atlas 2012, Holm et al. 2016). Briefly, the

Published by Bioscientifica Ltd. 
gene-normalized RSEM count estimates were offset by a pseudocount of $1, \log 2$-transformed and mean centered across tumor samples to generate relative gene expression levels for 20,531 genes. Although the distribution of age and ER status were similar between cohort 3 and cohort 2 , cohort 3 was enriched for patients with positive nodes and larger tumors (Supplementary Table 1). The median follow-up for OS was 2.2 (range 0.1-19) years.

\section{Measurement of serum 27-hydroxycholesterol}

The concentration of $27 \mathrm{HC}$ in the pre- and poststatin-treated serum samples were determined by mass spectrometry, as previously described, with modifications to improve throughput and specificity (Ayciriex et al. 2012). The LipidMaps standard cholest-5-ene-3ß,27-diol (Product 110818, Avanti Polar Lipids, Alabaster, AL, USA) was used to generate a standard curve in $50 \mathrm{mg} / \mathrm{mL}$ BSA matrix at final concentrations between $0.05 \mu \mathrm{M}$ and $10 \mu \mathrm{M}$. $50 \mu \mathrm{L}$ of serum, calibration standard or quality control (QC) sample was added to a 96-well plate, spiked with $1 \mu \mathrm{M}$ d6-27 HC internal standard in $\mathrm{MeOH}$ (Product LM-4114, Avanti Polar Lipids), followed by saponification with ethanolic sodium hydroxide. Saponified material was extracted with hexanes, derivatized with 4-(dimethylamino)phenyl isocyanate (Sigma-Aldrich) in dimethylformamide and triethylamine, quenched with phosphate buffer and re-extracted with hexanes. Extracts were then dried under nitrogen gas and reconstituted in 4:3:1 IPA:MeCN:H2O. 27HC was measured by a targeted UPLC-MS/MS (Xevo TQS, Waters) method monitoring the MRM transitions was quantified by calculating the ratio to internal standard, followed by linear regression with $1 / \mathrm{x}$ weighting against the calibration curve in Skyline v3.5.1.9942. Quantitative values were reported in micromolar concentrations. Low and high QC at 0.25 and $2.5 \mu \mathrm{M}$ showed accuracy of $109 \%$ and $98 \%$, respectively over three determinations.

\section{CYP27A1 protein expression (immunohistochemistry)}

The tumor cell-specific expression of CYP27A1 was determined by immunohistochemistry following a previously validated protocol (Nelson et al. 2013). Sections of 3 to $4 \mu \mathrm{m}$ were cut from whole tissue FFPE blocks, de-paraffinized, treated with antigen retrieval buffer (citrate, $\mathrm{pH}$ 6) for $20 \mathrm{~min}$, and then reacted with an anti-CYP27A1 rabbit monoclonal antibody (ab126785, Abcam) at a dilution of 1:500 for $2 \mathrm{~h}$. Staining procedures were performed using the DAKO Envision horseradish peroxidase rabbit/mouse kit (DAKO) and the Dakocytomation Autostainer (DAKO). Cell nuclei were counterstained with hematoxylin. CYP27A1 positivity was detected as a granular cytoplasmic reactivity. Cell type identification and staining intensity was assessed by a board certified pathologist (DG). Only tumor cell-specific CYP27A1 expression was considered for subsequent analyses. Each sample was given a semiquantitative intensity score: 0 (absent), 0.5 (borderline), 1 (weak), 2 (moderate) or 3 (strong). For statistical analysis, the tumors were categorized as negative (0), weak $(0.5$, $1)$ and overexpressed $(2,3)$. Representative cases of CYP27A1 expression in these categories are illustrated in Supplementary Fig. 4.

\section{Statistical analyses}

Associations between serum 27HC at baseline, posttreatment or the statin-induced change in serum 27HC (post-treatment level minus pre-treatment level) with other baseline tumor pathological factors and the statininduced change in tumor proliferation were assessed using the Mann-Whitney $U$ test for variables with 2 categories and Spearman rho's test for variables with $n \geq 3$ ordered categories. The Wilcoxon signed-rank test was applied to assess the differences in the distribution of CYP27A1 expression in relation to statin treatment, and Fisher's exact tests were used to assess the associations between CYP27A1 expression and other tumor pathological factors (cohort 1). To test for associations between CYP27A1 expression and other conventional primary tumor pathological factors (cohorts 2 and 3 ), the median expression of CYP27A1 was used to categorize patients into two groups (high; $\geq$ median and low; $<$ median), and chi-squared tests were implemented to identify statistically significant associations. Kaplan-Meier plots were generated to visualize the survival difference between patients with low vs high CYP27A1-expressing tumors and the log-rank test was used to check for significance. Cox proportional hazards models were used to evaluate if CYP27A1 expression provided independent prognostic information beyond the conventional prognostic factors in primary breast cancer including the age at primary tumor diagnosis, nodal status, histological grade, tumor size and hormone receptor status. $P$ values from two-sided statistical tests are reported and $P<0.05$ was considered to be significant. 


\section{Results}

\section{Associations between serum 27HC and other serum lipids and primary tumor pathological characteristics}

Initially, the distribution and relationship between $27 \mathrm{HC}$ and three main serum lipids: total cholesterol, LDL cholesterol and HDL cholesterol, were investigated. Fortytwo patients from cohort 1 were eligible for inclusion in these analyses. Of these, $83 \%$ were aged above 50 years and only $12 \%$ presented with $\mathrm{ER} \alpha$ negative tumors, indicating homogeneity of the cohort in relation to menopausal status and hormone receptor expression. Baseline concentrations of total cholesterol (mean; $6.05 \mathrm{mmol} / \mathrm{L}$, range; 4.4$9.9 \mathrm{mmol} / \mathrm{L}$ ), LDL cholesterol (mean: $3.72 \mathrm{mmol} / \mathrm{L}$, range; $2.26-6.85 \mathrm{mmol} / \mathrm{L}$ ) and HDL cholesterol (mean; $1.6 \mathrm{mmol} / \mathrm{L}$, range; $1.0-2.8 \mathrm{mmol} / \mathrm{L}$ ) were predominantly within the clinical 'normal' reference ranges defined for cardiovascular disease risk assessment, exemplifying another degree of homogeneity within this cohort.

The mean baseline concentration of 27HC in this cohort was $0.31 \mu \mathrm{M}$ (range $0.15-0.63 \mu \mathrm{M}$ ). Serum 27HC was found to be significantly positively correlated with total cholesterol (Fig. 1A) and LDL cholesterol levels (Fig. 1B), but not with HDL cholesterol (Fig. 1C). No statistically significant association was found between baseline serum 27HC concentration and primary tumor pathological factors including ER $\alpha$ status, tumor size, histological grade, tumor proliferation status (Ki67) and tumor cell-specific CYP27A1 expression (Table $1, P>0.05$ for all comparisons).

\section{Effects of statin treatment on serum 27HC and CYP27A1 expression in primary breast tumors}

Several studies in the past have suggested an association between serum $27 \mathrm{HC}$ and total cholesterol although the impact of statin-dependent lowering of cholesterol on
27HC levels has never been established. In this study, statin treatment was found to effectively decrease serum total cholesterol and LDL cholesterol, and this was accompanied by a commensurate decreased in 27HC concentrations in all patients. HDL cholesterol was however not significantly altered by the treatment (Fig. $2 \mathrm{~A})$. Noteworthy, the absolute reduction in $27 \mathrm{HC}$ levels were relatively smaller than the absolute decrease in total cholesterol and LDL cholesterol levels. The reduction in serum $27 \mathrm{HC}$ was not associated with the previously established anti-proliferative response to statin treatment in this cohort, measured as a change in the expression of the proliferation marker Ki67 (Bjarnadottir et al. 2013) (Table $1, P=0.82$ ).

Having established that statin treatment effectively decreased serum $27 \mathrm{HC}$, we next investigated if statins influenced CYP27A1 expression, at the protein and mRNA level, in breast tumors. A non-significant decrease in CYP27A1 mRNA expression after statin treatment (Fig. 2B, $P=0.09)$ was noted. In contrast, CYP27A1 protein expression was found to be significantly upregulated $(P=0.033$, Fig. 2C) following statin treatment. Regardless, the anti-proliferative effect of statin treatment (change in Ki67 index) was neither significantly correlated with changes in serum 27HC (Table $1, P=0.82$ ) nor to changes in intratumoral CYP27A1 protein expression (Supplementary Table 2, $P=0.33$ ). Further, the baseline and statin-induced changes in 27HC and CYP27A1 protein expression were not significantly associated with any other baseline tumor pathological factor. However, an association approaching statistical significance $(P=0.044)$ was observed between high tumor proliferation and high CYP27A1 expression (Table 1 and Supplementary Table 2 , respectively). These data indicate that although statins effectively decrease serum 27HC, and may alter CYP27A1 protein expression in breast tumors, the baseline or
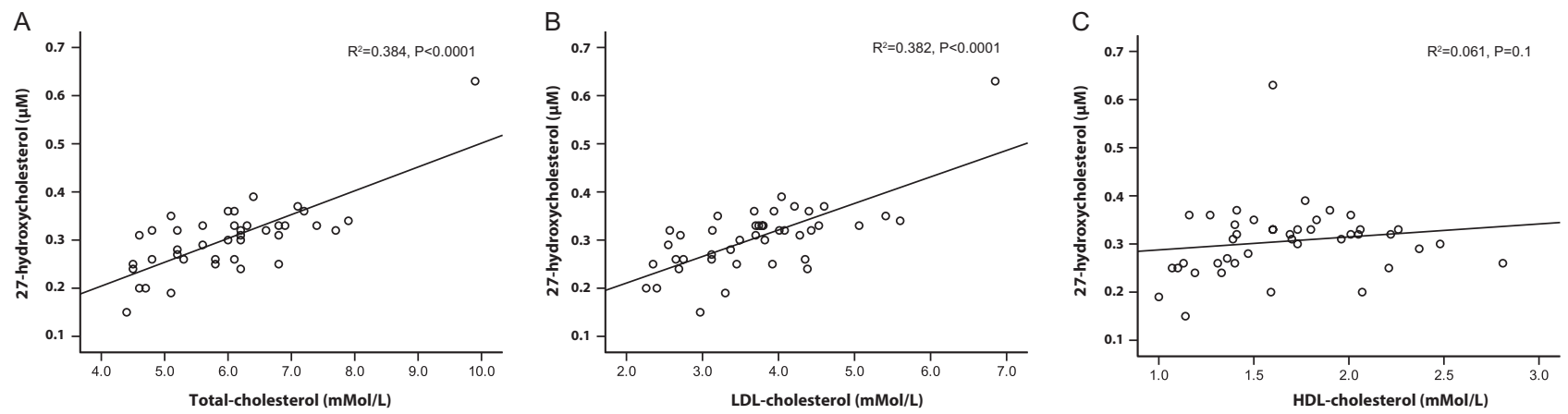

Figure 1

Relationship between $27 \mathrm{HC}$ and total cholesterol (A), LDL cholesterol (B) and HDL cholesterol (C) in serum of breast cancer patients before statin treatment (cohort 1). $P$ values are from Spearman's rho correlation tests. 
Table 1 Association between breast cancer tumor pathological features and serum 27HC concentrations in cohort 1. Correlations between baseline tumor pathological factors and $27 \mathrm{HC}$ levels before atorvastatin, post atorvastatin and the change in $27 \mathrm{HC}$ concentration following atorvastatin treatment were studied separately.

\begin{tabular}{|c|c|c|c|c|c|c|c|}
\hline \multirow[b]{2}{*}{ Factor } & \multicolumn{3}{|c|}{ 27HC Pre-atorvastatin } & \multicolumn{2}{|c|}{ 27HC Post-atorvastatin } & \multicolumn{2}{|l|}{ Change in 27HC* } \\
\hline & $n$ & Mean; $\mu \mathrm{M}(95 \% \mathrm{Cl})$ & $P$ & Mean; $\mu \mathrm{M}(95 \% \mathrm{Cl})$ & $P$ & Mean post-pre; $\mu \mathrm{M}(95 \% \mathrm{Cl})$ & $P$ \\
\hline \multicolumn{8}{|l|}{ Tumor size } \\
\hline$\leq 20 \mathrm{~mm}$ & 20 & $0.29(0.26-0.32)$ & 0.23 & $0.22(0.19-0.23)$ & 0.22 & $-0.07(-0.09,-0.05)$ & 0.18 \\
\hline$>20 \mathrm{~mm}$ & 22 & $0.32(0.29-0.36)$ & & $0.24(0.22-0.26)$ & & $-0.09(-0.10,-0.07)$ & \\
\hline \multicolumn{8}{|l|}{ Estrogen receptor } \\
\hline Positive & 37 & $0.30(0.27-0.33)$ & 0.34 & $0.23(0.21-0.24)$ & 0.36 & $-0.08(-0.09,-0.07)$ & 1.0 \\
\hline Negative & 5 & $0.32(0.21-0.41)$ & & $0.24(0.19-0.30)$ & & $-0.07(-0.13,-0.02)$ & \\
\hline \multicolumn{8}{|l|}{ Histological grade } \\
\hline 1 & 9 & $0.31(0.27-0.35)$ & 0.44 & $0.21(0.18-0.24)$ & 0.50 & $-0.10(-0.14,-0.07)$ & 0.10 \\
\hline 2 & 17 & $0.31(0.26-0.36)$ & & $0.24(0.20-0.27)$ & & $-0.08(-0.10,-0.06)$ & \\
\hline 3 & 16 & $0.29(0.26-0.33)$ & & $0.23(0.21-0.25)$ & & $-0.07(-0.09,-0.05)$ & \\
\hline \multicolumn{8}{|l|}{ Ki67 } \\
\hline$\leq 20 \%$ & 19 & $0.32(0.27-0.26)$ & 0.52 & $0.23(0.20-0.26)$ & 0.73 & $-0.09(-0.11,-0.07)$ & 0.39 \\
\hline$>20 \%$ & 15 & $0.29(0.25-0.32)$ & & $0.22(0.19-0.24)$ & & $-0.07(-0.09,-0.05)$ & \\
\hline \multicolumn{8}{|l|}{ CYP27A1 } \\
\hline Negative & 7 & $0.30(0.25-0.34)$ & 0.81 & $0.21(0.19-0.23)$ & 0.72 & $-0.08(-0.11,-0.06)$ & 0.45 \\
\hline Borderline / Weak & 15 & $0.32(0.27-0.37)$ & & $0.24(0.20-0.27)$ & & $-0.08(-0.11,-0.05)$ & \\
\hline Moderate / Strong & 4 & $0.27(0.19-0.36)$ & & $0.21(0.17-0.25)$ & & $-0.06(-0.11,-0.01)$ & \\
\hline \multicolumn{8}{|l|}{ Change in Ki67 index* } \\
\hline Decreased & 20 & $0.29(0.27-0.32)$ & $P=0.87$ & $0.22(0.20-0.23)$ & $P=1.0$ & $-0.08(-0.10,-0.06)$ & $P=0.82$ \\
\hline Increased & 14 & $0.32(0.26-0.37)$ & & $0.23(0.20-0.27)$ & & $-0.08(-1.03,-0.06)$ & \\
\hline
\end{tabular}

$P$-values from Mann-Whitney $U$ test for variables with 2 categories and Spearman rho's test for variables with $n \geq 3$ ordered categories *Effect of statin treatment expressed as the difference between the post-treatment and pre-treatment level.

statin-induced changes in 27HC or CYP27A1 do not appear to explain the anti-proliferative response to statin treatment seen in the tumors in this cohort.

\section{Associations between CYP27A1 expression and baseline primary breast tumor pathological characteristics and prognosis}

In preclinical models, it has been shown that in addition to statins, $27 \mathrm{HC}$ levels can be lowered by direct inhibition of CYP27A1 (Nelson et al. 2013). Thus, this enzyme may be a useful drug target in breast cancer. This far, however, the expression of CYP27A1 in breast tumors has only been evaluated in small, underpowered studies. Consequently, we explored how variability in CYP27A1 expression, measured both at the transcriptional and protein levels, may influence breast cancer pathology and prognosis. Differential CYP27A1 mRNA expression was found to be significantly associated with breast cancer tumor pathological factors. In cohort 2, high CYP27A1
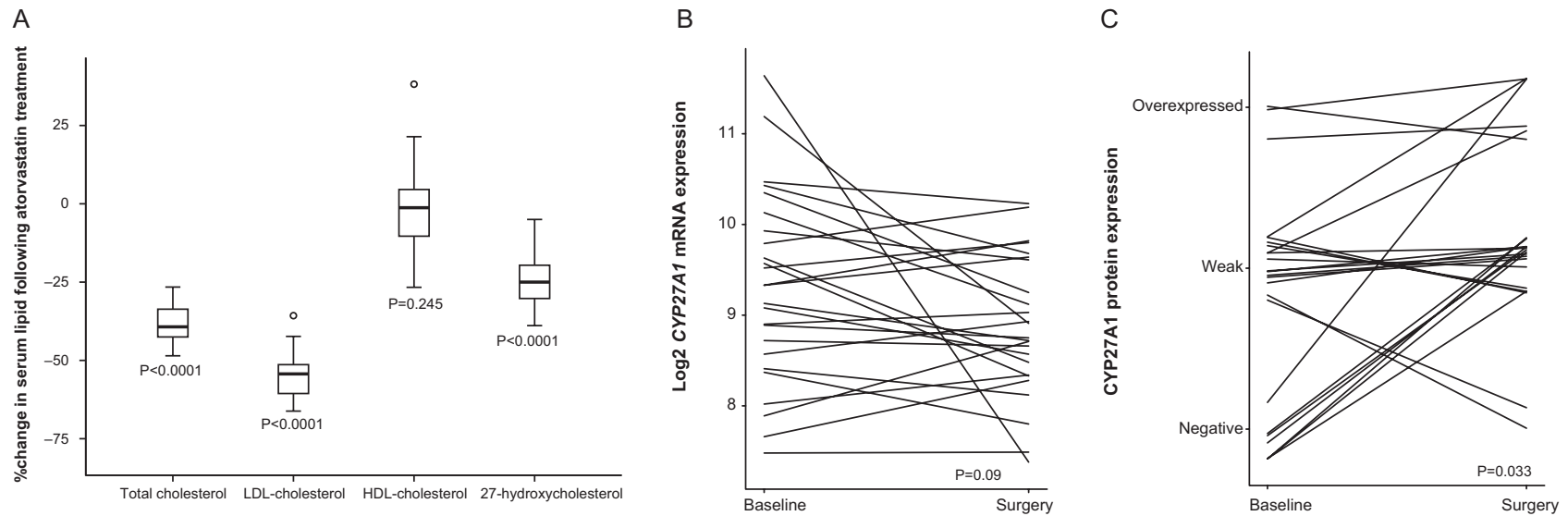

Figure 2

Statin-induced changes in serum lipids (A) and CYP27A1 mRNA expression in tumors (B) and tumor cell specific CYP27A1 protein expression (C). $P$ values are from the Wilcoxon signed-rank test comparing the pre-treatment (baseline) and post-treatment (surgery) measurement for each factor. 
expression was more frequent in tumors among younger women (age $\leq 50$ years), and in node-negative, ER $\alpha$ negative, high-grade (grade 3), basal-like and normal-like breast cancer (Table 2). A similar association between age and nodal status with CYP27A1 expression was observed in sub-analyses that considered ERo-positive tumors alone (Table 2). Furthermore, ER $\alpha$ negativity, negative nodal status and the basal-like and normal-like molecular subtypes were confirmed to be associated with high CYP27A1 expression in the TCGA cohort (Table 2).

Next, we investigated if CYP27A1 expression may predict primary breast cancer prognosis. In an analysis that included all patients within cohort 2, a marginal trend toward a better RFS was observed for patients presenting with high CYP27A1 expression in the primary tumor $(\mathrm{HR}=0.82, \mathrm{CI}=0.66-1.02, P=0.07$, Supplementary Fig. 1A). This relationship was maintained when only the subset of ER $\alpha$-positive tumors were considered $(\mathrm{HR}=0.76$, $\mathrm{CI}=0.59-0.98, P=0.04$, Fig. 3A). Interestingly, when the analysis was further stratified for age at diagnosis, the prolonged RFS associated with high CYP27A1 expression was found to be limited to the subgroup of women $\leq 50$ years of age (Fig. 3B and C). The predictive value of CYP27A1 expression for OS was similar to RFS (Fig. 3D, E and F and Supplementary Fig. 1B). High CYP27A1 expression was independently prognostic for longer RFS and OS in multivariable analyses, especially among younger women (Table 3). Independent analyses in the TCGA cohort confirmed that high CYP27A1 expression was associated with prolonged overall survival (Supplementary Fig. 2A, $\mathrm{HR}=0.49, \mathrm{CI}=0.34-0.78, P=0.003)$, and this relationship remained significant for analyses that included only those tumors that were ER $\alpha$ positive (Supplementary Fig. 2B, $\mathrm{HR}=0.51, \mathrm{CI}=0.28-0.94, P=0.03)$. Further stratification of ERo-positive tumors within the TCGA cohort based on age suggested that high expression of CYP27A1 was associated with better overall survival in both age groups, but the results were not statistically significant for the subgroup of women under 50 years of age (Supplementary Fig. 2C and D).

Finally, the prognostic relevance of CYP27A1 in ER $\alpha$ negative breast cancers was investigated. Although high CYP27A1 expression was more frequently observed among ER $\alpha$-negative tumors, no significant difference in RFS and OS was seen between high and low CYP27A1 expressing ER $\alpha$-negative tumors in cohort 2 (Supplementary Fig. 3A and B). In contrast, a similar analysis in the TCGA cohort resulted in a statistically significant difference favoring a prolonged OS among high CYP27A1-expressing ER $\alpha$ negative tumors relative to low CYP27A1-expressing $\mathrm{ER} \alpha$-negative tumors (Supplementary Fig. 3C, HR=0.41, $\mathrm{CI}=0.18-0.92, P=0.03)$.

Table 2 Associations between primary breast cancer tumor pathological factors and CYP27A1 mRNA.

\begin{tabular}{|c|c|c|c|c|c|c|c|c|c|}
\hline \multirow[b]{2}{*}{ Factor at baseline } & \multicolumn{3}{|c|}{ CYP27A1 (all cohort 2) } & \multicolumn{3}{|c|}{ CYP27A1 (ER-positive cohort 2) } & \multicolumn{3}{|c|}{ CYP27A1 (Cohort 3) } \\
\hline & Low (\%) & High (\%) & $P$ & Low (\%) & High (\%) & $P$ & Low (\%) & High (\%) & $P$ \\
\hline \multicolumn{10}{|l|}{ Age at diagnosis } \\
\hline$\leq 50$ years & 47 & 53 & $<0.001$ & 50 & 50 & 0.002 & 52 & 48 & 0.56 \\
\hline$>50$ years & 58 & 42 & & 60 & 40 & & 49 & 51 & \\
\hline \multicolumn{10}{|l|}{ Tumor size } \\
\hline$\leq 20 \mathrm{~mm}$ & 48 & 52 & 0.29 & 54 & 46 & 0.08 & 47 & 53 & 0.25 \\
\hline$>20 \mathrm{~mm}$ & 51 & 49 & & 59 & 41 & & 52 & 48 & \\
\hline \multicolumn{10}{|l|}{ Nodal status } \\
\hline Positive & 67 & 33 & $<0.001$ & 68 & 32 & $<0.001$ & 53 & 47 & 0.047 \\
\hline Negative & 44 & 56 & & 51 & 49 & & 46 & 54 & \\
\hline \multicolumn{10}{|c|}{ Estrogen receptor status } \\
\hline Positive & 55 & 45 & 0.001 & n.a & n.a & n.a & 54 & 46 & 0.001 \\
\hline Negative & 46 & 54 & & & & & 38 & 62 & \\
\hline \multicolumn{10}{|l|}{ Molecular subtype } \\
\hline Luminal A & 53 & 47 & & 56 & 44 & & 52 & 48 & \\
\hline Luminal B & 54 & 46 & & 57 & 43 & & 67 & 33 & \\
\hline Her2-enriched & 65 & 35 & $<0.001$ & 68 & 32 & $<0.001$ & 57 & 43 & $<0.001$ \\
\hline Basal-like & 32 & 67 & & 32 & 68 & & 31 & 69 & \\
\hline Normal-like & 44 & 56 & & 41 & 51 & & 34 & 66 & \\
\hline \multicolumn{10}{|l|}{ Histological grade } \\
\hline 1 and 2 & 54 & 46 & $<0.001$ & 60 & 40 & 0.003 & n.a & n.a & n.a \\
\hline 3 & 42 & 58 & & 50 & 50 & & & & \\
\hline
\end{tabular}

$P$-values from Pearson Chi-Square test. n.a; not applicable. Values in bold represent statistically significant $P$ values.

http://erc.endocrinology-journals.org DOI: 10.1530/ERC-16-0533
(C) 2017 The authors Printed in Great Britain
Published by Bioscientifica Ltd 
A

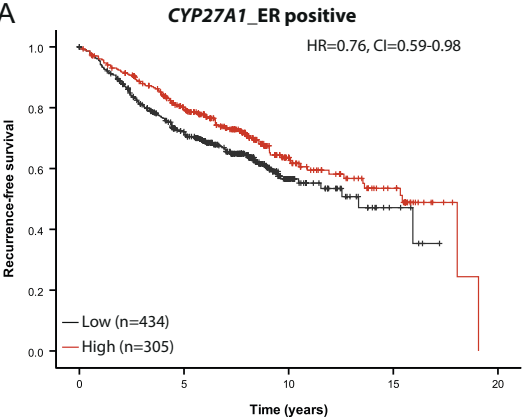

D

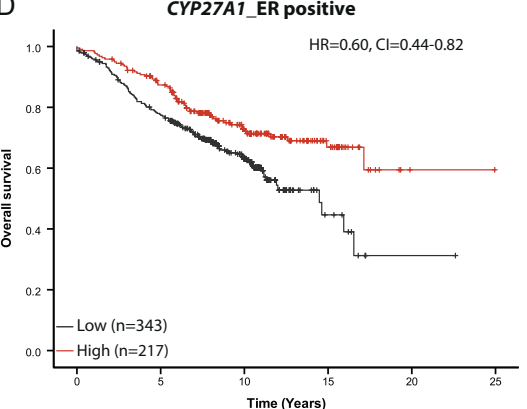

B

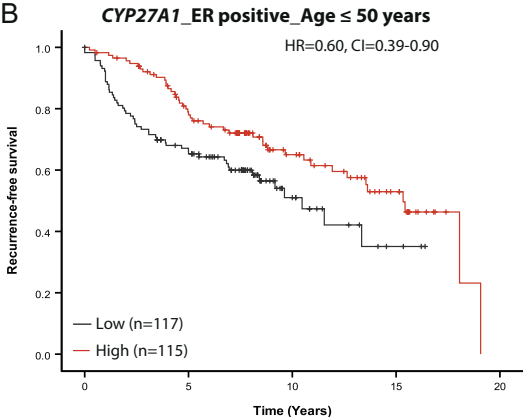

E

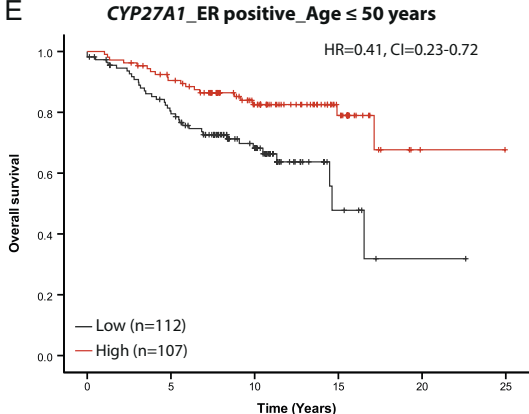

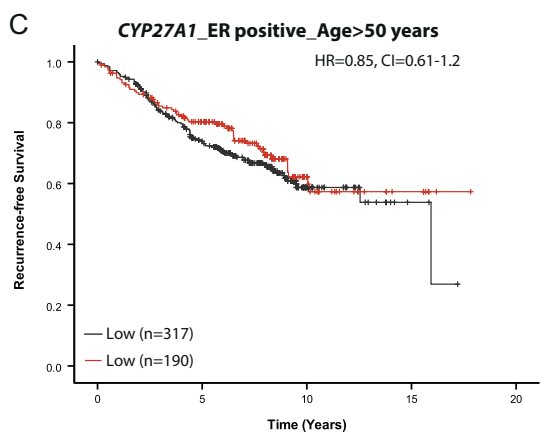

$\mathrm{F}$

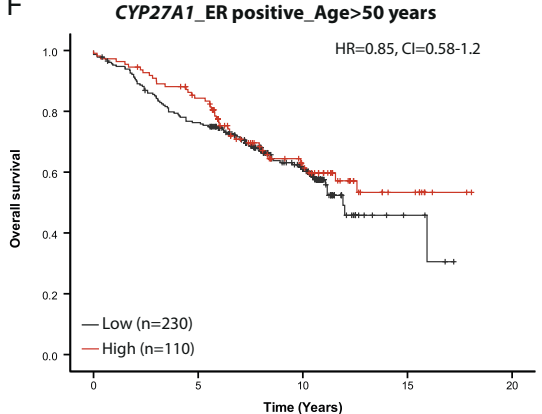

Figure 3

Associations between CYP27A1 expression and survival in estrogen receptor-positive primary breast cancer (cohort 2). High expression of CYP27A1 is associated with a longer recurrence-free survival, RFS (A-C) and overall survival, OS (D-F) in ER $\alpha$ positive breast cancer.

\section{Discussion}

The results of studies from our laboratories and others have suggested that hypercholesterolemia, secondary to its conversion to $27 \mathrm{HC}$ by CYP27A1, may impact breast cancer recurrence risk by activating ER $\alpha$. It was inferred therefore that increased circulating $27 \mathrm{HC}$ and/or CYP27A1 would be associated with poorer outcomes in patients with $\mathrm{ER} \alpha$-positive tumors and that this risk could be mitigated by statins. Leveraging these findings we developed a clinical study to (a) explore the relationship between cholesterol and circulating 27HC, (b) define the impact of statins on 27HC levels and (c) define the prognostic significance of $27 \mathrm{HC}$ and/or intratumoral CYP27A1 on tumor pathobiology. It was anticipated that such a study would inform the future development of a prospective adjuvant clinical trial to establish the role of lipid-lowering medications like statins in breast cancer treatment.

The results from small early-phase pre-surgical (Garwood et al. 2010) and biomarker discovery trials (Higgins et al. 2012), including the results from the window-of-opportunity trial (Bjarnadottir et al. 2013) presented herein, have unanimously confirmed that the compliance to statin treatment is remarkably high and that these drugs effectively decrease serum total cholesterol and LDL cholesterol. Further, in this study, we have shown for the first time that statin-induced reduction in serum cholesterol is accompanied by a corresponding decrease in serum 27HC. This finding afforded us the opportunity to explore the relationship, if any, between the beneficial effects of statins on tumor biology, noted previously, and

Table 3 Association between CYP27A1 expression and prognosis following primary breast cancer diagnosis in cohort 2.

\begin{tabular}{|c|c|c|c|c|c|c|}
\hline \multirow[b]{2}{*}{ Patient category } & \multicolumn{3}{|c|}{ RFS_CYP27A1 (ref. low) } & \multicolumn{3}{|c|}{ OS_CYP27A1 (ref. low) } \\
\hline & $\mathrm{HR}$ & $\mathrm{Cl}$ & $P$ & $\mathrm{HR}$ & $\mathrm{Cl}$ & $P$ \\
\hline All tumors & 0.76 & $0.60-0.96$ & 0.02 & 0.82 & $0.62-1.1$ & 0.16 \\
\hline All ER positive tumors & 0.70 & $0.53-0.92$ & 0.01 & 0.74 & $0.53-1.0$ & 0.07 \\
\hline ER positive/ $\leq 50$ years & 0.53 & $0-33-0.83$ & 0.01 & 0.49 & $0.27-0.89$ & 0.02 \\
\hline ER Positive/>50 years & 0.85 & $0.60-1.2$ & 0.36 & 0.93 & $0.62-1.4$ & 0.7 \\
\hline
\end{tabular}

Multivariable Cox-regression analyses for recurrence-free survival (RFS) and overall survival (OS). $P<0.05$ was considered significant.

Adjusted for age at diagnosis, ER status, nodal status, histological grade and tumor size where applicable. HR; hazard ratio, Cl; confidence interval, RFS; recurrence-free survival, OS; overall survival, ref; reference, ER; estrogen receptor. Values in bold represent statistically significant $P$ values.

http://erc.endocrinology-journals.org

DOI: 10.1530/ERC-16-0533
(C) 2017 The authors Printed in Great Britain
Published by Bioscientifica Ltd. 
$27 \mathrm{HC}$ production. However, baseline serum $27 \mathrm{HC}$ and the magnitude of the treatment-induced reduction in serum 27HC were neither associated with any baseline tumor pathological factor nor the anti-proliferative tumor response to statin treatment in the 42 patient cohort included in this study.

Irrespective of how it was analyzed (mRNA or protein), the change in CYP27A1 was also not associated with any baseline tumor pathological factor nor did it correlate with the anti-proliferative tumor response to statins. These results suggest that the efficacy of statin treatment in controlling breast cancer proliferation cannot be predicted from serum $27 \mathrm{HC}$ and that tumor cell-specific CYP27A1 expression does not inform the likely tumor response to statin treatment. However, the homogeneity within this cohort with respect to basal levels of serum lipids and patient and tumor pathological factors like age of study participants and ER $\alpha$ status, coupled with the small numbers of patients included in the study, may have limited the power to detect any statistically significant associations in our analyses.

Although statins effectively lower circulating cholesterol, their impact on intratumoral cholesterol content/production is likely to be less substantial. This is due in part to variation in the post-hepatic exposure level of these drugs and also to compensatory upregulation of cholesterol synthesizing enzymes and uptake machinery and decreased cholesterol efflux in cells in response to low cholesterol. Thus, significant reduction of intratumoral production of $27 \mathrm{HC}$ may not be accomplished by reducing circulating cholesterol. Small-molecule inhibitors of CYP27A1 have been shown to effectively reduce both circulating and intratumoral $27 \mathrm{HC}$ and may represent an adjunct to statins to mitigate the impact of this oxysterol in breast cancer. Interestingly, the aromatase inhibitor anastrozole was recently shown to be a potent 'off-target' inhibitor of CYP27A1 that significantly reduces plasma $27 \mathrm{HC}$ without affecting hepatic and plasma cholesterol levels (Mast et al. 2015). Considering these findings, it was of interest to evaluate the relationship between intratumoral CYP27A1 and pathological features of importance in breast cancer. Using immunohistochemistry, Nelson and coworkers (Nelson et al. 2013) reported that elevated expression of CYP27A1 was more frequently observed among high-grade tumors. Similarly, in this study, immunohistochemical analyses indicated that CYP27A1 was expressed in up to $91 \%$ of high-grade (grade 3 ) tumors compared to $60 \%$ of low-grade (grades 1 and 2) tumors, but this comparison did not reach statistical significance in this small cohort. To address this further, we made use of well-annotated publicly available transcriptional databases for breast cancer and probed the association between CYP27A1 expression and selected pathological factors in two independent gene expression cohorts. While confirming that high tumor grade was significantly associated with high CYP27A1 expression, we also determined that ER $\alpha$ negativity, basal-like and normal-like molecular subtypes were features that were associated with high CYP27A1 expression in both primary breast tumors and in breast cancer cell lines (data not shown).

The prognostic relevance of CYP27A1 expression in breast cancer has previously been investigated with null findings (Nelson et al. 2013, Wu et al. 2013). Interestingly, in two sizable, independent breast cancer cohorts we found, especially in ERo-positive disease, that CYP27A1 expression in the primary tumor provided independent prognostic information beyond conventional prognostic factors. Given that 27HC may perform conflicting roles in hypo- and normo/hyper-estrogenic conditions, it is important to establish if the prognostic importance of CYP27A1 is influenced by menopausal status. Notable was the observation that both RFS and OS were improved in patients aged less than 50 years presenting with ER $\alpha$-positive disease whose tumors expressed elevated CYP27A1. This could be explained by the fact that $27 \mathrm{HC}$, being a SERM, has the potential of reducing estradiolactivated ER $\alpha$ to a level equivalent to its inherent partial agonist activity. Thus, in normal cycling women, it would be expected that 27HC would attenuate the actions of estradiol. Further, it has to be noted that the normal physiological function of CYP27A1 is to regulate intracellular cholesterol homeostasis by converting cholesterol to the more polar 27HC and also potentially to the bile acid precursor cholestenoic acid. 27HC itself can function as a ligand of the liver $\mathrm{X}$ receptor and induce the expression of the cholesterol efflux pumps ABCA1 and ABCG1. In addition, 27HC can bind to INSIG1 and interfere with the activation of SREBP2, the master regulator of the expression of the enzymes required for cholesterol biosynthesis. Thus, under normal physiological circumstances, CYP27A1 and 27HC may actually exhibit a protective effect in breast cancer by limiting the ability of the cell to accumulate cholesterol. Interestingly, the protective effect of high CYP27A1 expression was lost in older women (presumably postmenopausal) indicating that high 27HC, consequent to high CYP27A1 expression, may play a pro-tumorigenic role in postmenopausal 
women and that decreasing $27 \mathrm{HC}$ by direct inhibition of CYP27A1 or indirectly with statin treatment may prolong survival in postmenopausal women with breast cancer. This result was however not verified in the TCGA cohort, but it is worth noting that the event rate (breast cancer deaths) was relatively lower in this cohort due to the limited follow-up time, the median of which was only 2.2 years, which is a time interval when more than $95 \%$ of patients with ERo-positive breast cancer are event-free after surgery and standard adjuvant therapy.

There are noteworthy caveats to this study. Restricted by insufficient tumor material, $27 \mathrm{HC}$ measurements were not performed for tumor samples; hence, we could not evaluate if a commensurate decrease in intratumoral 27HC levels occurs following statin treatment. The survival data in cohort 2 are derived from 11 datasets, comprising a very large cohort of nearly 2000 tumors that were heterogeneously treated. The fact that modern treatment has changed the natural history of breast cancer suggests that the prognostic implications of CYP27A1 detected by our analyses may represent historical behavior. Although most of the results were validated in the more recent TCGA cohort, the immature follow-up data limited our investigations.

\section{Conclusions}

In summary, our study provides new insights into the pathobiological and prognostic relevance of CYP27A1 expression in breast cancer. For the first time, we have shown that statin treatment effectively decreases serum 27HC and may deregulate CYP27A1 expression in tumors, although these changes do not correlate with the antiproliferative response of the treatment. Furthermore, our results indicate that CYP27A1 is differentially expressed in breast cancer with important consequences on the disease phenotype and prognosis. These results can have implications for selecting patients who may benefit from the addition of statins or drugs inhibiting CYP27A1 to their standard breast cancer therapeutic regimen. Our results also highlight the need for further studies to evaluate how tissue-specific and/or systemic levels of 27HC and CYP27A1 impact breast cancer pathobiology and response to endocrine and statin treatment in additional cohorts representing the clinical disease.

\section{Supplementary data}

This is linked to the online version of the paper at http://dx.doi.org/10.1530/ ERC-16-0533.
Declaration of interest

$S$ Borgquist has received consultant and speaker fees from Roche and Novartis. The other authors declare that they have no competing interests.

\section{Funding}

This work was supported by grants from the Governmental Funding of Clinical Research from the National Health Services, the Swedish Breast Cancer Organization (BRO), the Mrs Berta Kamprad Foundation, the Swedish Research Council and the Swedish Cancer Foundation.

\section{Authors' contribution statement}

Drs Kimbung and Borgquist had full access to all of the data in the study and take responsibility for the integrity of the data and the accuracy of the data analysis. Study Concept and Design: Kimbung, Chang, McDonnell, Borgquist. Acquisition of data: Kimbung, Dubois, Thompson, Borgquist. Analysis and interpretation of data: Kimbung, Chang, Bendahl, Dubois, Thompson, McDonnell, Borgquist. Drafting of the manuscript: Kimbung, Chang, McDonnell, Borgquist. Critical revision of the manuscript for important intellectual content: Kimbung, Chang, Bendahl, Dubois, Thompson, McDonnell, Borgquist. Statistical analysis: Kimbung, Bendahl. Obtained funding: Borgquist. Administrative, technical, or material support: Dubois, Thompson, McDonnell, Borgquist. Study supervision: Kimbung, Chang, McDonnell, Borgquist. All authors read and approved the final manuscript.

\section{Acknowledgements}

The authors express their profound gratitude to Kristina Lövgren for performing the CYP27A1 IHC staining. They are also very highly indebted to the Senior Pathologist Dorthe Grabau+ for annotating of the CYP27A1 IHC slides.

\section{References}

Ahern TP, Pedersen L, Tarp M, Cronin-Fenton DP, Garne JP, Silliman RA, Sorensen HT \& Lash TL 2011 Statin prescriptions and breast cancer recurrence risk: a Danish nationwide prospective cohort study. Journal of the National Cancer Institute 103 1461-1468. (doi:10.1093/ jnci/djr291

Ayciriex S, Regazzetti A, Gaudin M, Prost E, Dargere D, Massicot F, Auzeil N \& Laprevote O 2012 Development of a novel method for quantification of sterols and oxysterols by UPLC-ESI-HRMS: application to a neuroinflammation rat model. Analytical and Bioanalytical Chemistry 404 3049-3059. (doi:10.1007/s00216-0126396-6)

Bianchini F, Kaaks R \& Vainio H 2002 Overweight, obesity, and cancer risk. Lancet Oncology 3 565-574. (doi:10.1016/S1470-2045(02)00849-5)

Bjarnadottir O, Kimbung S, Johansson I, Veerla S, Jonsson M, Bendahl PO, Grabau D, Hedenfalk I \& Borgquist S 2015 Global Transcriptional Changes Following Statin Treatment in Breast Cancer. Clinical Cancer Research 21 3402-3411. (doi:10.1158/10780432.CCR-14-1403)

Bjarnadottir O, Romero Q, Bendahl PO, Jirstrom K, Ryden L, Loman N, Uhlen M, Johannesson H, Rose C, Grabau D, et al. 2013 Targeting HMG-CoA reductase with statins in a window-of-opportunity breast cancer trial. Breast Cancer Research and Treatment 138 499-508. (doi:10.1007/s10549-013-2473-6)

Published by Bioscientifica Ltd http://erc.endocrinology-journals.org DOI: 10.1530/ERC-16-0533
(C) 2017 The authors Printed in Great Britain 
Borgquist S, Giobbie-Hurder A, Ahern TP, Garber JE, Colleoni M, Láng I, Debled M, Ejlertsen B, Coates AS, Goldhirsch A, et al. 2016 Cholesterol, cholesterol lowering medication use, and breast cancer outcomes in the BIG 1-98 study. Proceedings of the Thirty-Eighth Annual CTRC-AACR San Antonio Breast Cancer Symposium, Dec 8-12 2015. Cancer Research 76 (4 Suppl) abstract PD1-03.

Boudreau DM, Yu O, Chubak J, Wirtz HS, Bowles EJ, Fujii M \& Buist DS 2014 Comparative safety of cardiovascular medication use and breast cancer outcomes among women with early stage breast cancer. Breast Cancer Research and Treatment 144 405-416. (doi:10.1007/s10549014-2870-5)

Boyd NF \& Mcguire V 1990 Evidence of Association between Plasma High-Density-Lipoprotein Cholesterol and Risk-Factors for BreastCancer. Journal of the National Cancer Institute 82 460-468. (doi:10.1093/jnci/82.6.460)

Brown AJ \& Jessup W 1999 Oxysterols and atherosclerosis. Atherosclerosis 142 1-28. (doi:10.1016/S0021-9150(98)00196-8)

Burkard I, von Eckardstein A, Waeber G, Vollenweider P \& Rentsch KM 2007 Lipoprotein distribution and biological variation of 24S- and 27-hydroxycholesterol in healthy volunteers. Atherosclerosis 194 71-78. (doi:10.1016/j.atherosclerosis.2006.09.026)

Cancer Genome Atlas N 2012 Comprehensive molecular portraits of human breast tumours. Nature 490 61-70.

Chae YK, Valsecchi ME, Kim J, Bianchi AL, Khemasuwan D, Desai A \& Tester W 2011 Reduced risk of breast cancer recurrence in patients using ACE inhibitors, ARBs, and/or statins. Cancer Investigation 29 585-593. (doi:10.3109/07357907.2011.616252)

Clendening JW \& Penn LZ 2012 Targeting tumor cell metabolism with statins. Oncogene 31 4967-4978. (doi:10.1038/onc.2012.6)

DuSell CD, Umetani M, Shaul PW, Mangelsdorf DJ \& McDonnell DP 2008 27-hydroxycholesterol is an endogenous selective estrogen receptor modulator. Molecular Endocrinology 22 65-77. (doi:10.1210/ me.2007-0383)

Garwood ER, Kumar AS, Baehner FL, Moore DH, Au A, Hylton N, Flowers CI, Garber J, Lesnikoski BA, Hwang ES, et al. 2010 Fluvastatin reduces proliferation and increases apoptosis in women with high grade breast cancer. Breast Cancer Research and Treatment 119 137-144. (doi:10.1007/s10549-009-0507-x)

Higgins MJ, Prowell TM, Blackford AL, Byrne C, Khouri NF, Slater SA, Jeter SC, Armstrong DK, Davidson NE, Emens LA, et al. 2012 A short-term biomarker modulation study of simvastatin in women at increased risk of a new breast cancer. Breast Cancer Research and Treatment 131 915-924. (doi:10.1007/s10549-011-1858-7)

Holm K, Staaf J, Lauss M, Aine M, Lindgren D, Bendahl PO, VallonChristersson J, Barkardottir RB, Hoglund M, Borg A, et al. 2016 An integrated genomics analysis of epigenetic subtypes in human breast tumors links DNA methylation patterns to chromatin states in normal mammary cells. Breast Cancer Research 18 27. (doi:10.1186/ s13058-016-0685-5)

Kitahara CM, Berrington de Gonzalez A, Freedman ND, Huxley R, Mok Y, Jee SH \& Samet JM 2011 Total cholesterol and cancer risk in a large prospective study in Korea. Journal of Clinical Oncology 29 1592-1598. (doi:10.1200/JCO.2010.31.5200)

Mast N, Lin JB \& Pikuleva IA 2015 Marketed drugs can inhibit cytochrome P450 27A1, a potential new target for breast cancer adjuvant therapy. Molecular Pharmacology 88 428-436. (doi:10.1124/ mol.115.099598)

McDonnell DP, Park S, Goulet MT, Jasper J, Wardell SE, Chang CY, Norris JD, Guyton JR \& Nelson ER 2014 Obesity, cholesterol metabolism, and breast cancer pathogenesis. Cancer Research $\mathbf{7 4}$ 4976-4982. (doi:10.1158/0008-5472.CAN-14-1756)

Nelson ER, Wardell SE, Jasper JS, Park S, Suchindran S, Howe MK, Carver NJ, Pillai RV, Sullivan PM, Sondhi V, et al. 2013 27-Hydroxycholesterol links hypercholesterolemia and breast cancer pathophysiology. Science 342 1094-1098. (doi:10.1126/science.1241908)

Ringner M, Fredlund E, Hakkinen J, Borg A \& Staaf J 2011 GOBO: gene expression-based outcome for breast cancer online. PLoS One 6 e17911. (doi:10.1371/journal.pone.0017911)

Spampanato C, De Maria S, Sarnataro M, Giordano E, Zanfardino M, Baiano S, Carteni M \& Morelli F 2012 Simvastatin inhibits cancer cell growth by inducing apoptosis correlated to activation of Bax and down-regulation of BCL-2 gene expression. International Journal of Oncology 40 935-941.

Umetani M, Domoto H, Gormley AK, Yuhanna IS, Cummins CL, Javitt NB, Korach KS, Shaul PW \& Mangelsdorf DJ 2007 27-Hydroxycholesterol is an endogenous SERM that inhibits the cardiovascular effects of estrogen. Nature Medicine 13 1185-1192. (doi:10.1038/nm1641)

Wu Q, Ishikawa T, Sirianni R, Tang H, McDonald JG, Yuhanna IS, Thompson B, Girard L, Mineo C, Brekken RA, et al. 2013 27-Hydroxycholesterol promotes cell-autonomous, ER-positive breast cancer growth. Cell Reports 5 637-645. (doi:10.1016/j. celrep.2013.10.006)

Received in final form 13 April 2017

Accepted 25 April 2017

Accepted Preprint published online 25 April 2017
ㄷ 2017 The authors Printed in Great Britain
Published by Bioscientifica Ltd. 